\title{
Logarithmic two dimensional spin-1/3 fractional supersymmetric conformal field theories and the two point functions
}

\author{
Fardin Kheirandish ${ }^{a}$ and Mohammad Khorrami ${ }^{a, b}$ F \\ ${ }^{a}$ Institute for Advanced Studies in Basic Sciences, \\ P. O. Box 159, Zanjan 45195, Iran \\ ${ }^{b}$ Institute for Studies in Theoretical physics and Mathematics, \\ P. O. Box 5531, Tehran 19395, Iran
}

\begin{abstract}
Logarithmic spin $-1 / 3$ superconformal field theories are investigated. The chiral and full two-point functions of two- (or more-) dimensional Jordanian blocks of arbitrary weights, are obtained.
\end{abstract}

\footnotetext{
1 e-mail:fardin@iasbs.ac.ir

2 e-mail:mamwad@iasbs.ac.ir
} 


\section{Introduction}

According to Gurarie [1], conformal field theories which their correlation functions exhibit logarithmic behaviour may be consistently defined. In some interesting physical theories like polymers [2], WZNW models [3-6], percolation [7], the Haldane-Rezayi quantum Hall state [8], and edge excitation in fractional quantum Hall effect [9], logarithmic correlation functions appear. Also the logarithmic operators can be considered in 2D-magnetohydrodynamic turbulence [10,11,12], 2D-turbulence ([13], [14]) and some critical disordered models [15,16]. Logarithmic conformal field theories for D dimensional case $(D>2)$ has also been studied [17]. In this paper we consider a superconformal extension of Virasoro algebra $[18,19]$ corresponding to three-component supermultiplets, and then, following [20-22], generalize the superconformal field to Jordanian blocks of quasi superconformal fields. We then find the two-point functions of chiraland full-component fields. It is seen that this correlators are readily obtained through formal derivatives of correlators of superprimary fields, just as was seen in $[20-22]$.

\section{Superprimary and quasi-superprimary fields}

A chiral superprimary field $\Phi(z, \theta)$ with conformal weight $\Delta$, is an operator satisfying [18]

$$
\begin{gathered}
{\left[L_{n}, \Phi(z, \theta)\right]=\left[z^{n+1} \partial_{z}+(n+1)\left(\Delta+\frac{\Lambda}{3}\right) z^{n}\right] \Phi(z, \theta),} \\
{\left[G_{r}, \Phi(z, \theta)\right]=\left[z^{r+1 / 3} \delta_{\theta}-q^{2} z^{r+1 / 3} \theta^{2} \partial_{z}-(3 r+1) q^{2} \Delta z^{r-2 / 3} \theta^{2}\right] \Phi(z, \theta),}
\end{gathered}
$$

where $\theta$ is a paragrassmann variable, satisfying $\theta^{3}=0, q$ is one of the third roots of unity, not equal to one, and $\delta_{\theta}$ and $\Lambda$, satisfy $[18,19]$

$$
\delta_{\theta} \theta=q^{-1} \theta \delta_{\theta}+1,
$$

and

$$
[\Lambda, \theta]=\theta, \quad\left[\Lambda, \delta_{\theta}\right]=-\delta_{\theta} .
$$

Here $L_{n}$ 's and $G_{r}$ 's are the generators of the supervirasoro algebra satisfying

$$
\begin{aligned}
{\left[L_{n}, L_{m}\right] } & =(n-m) L_{n+m} \\
{\left[L_{n}, G_{r}\right] } & =\left(\frac{n}{3}-r\right) G_{n+r}
\end{aligned}
$$

and

$$
G_{r} G_{s} G_{t}+\text { five other permutations of the indices }=6 L_{r+s+t} .
$$


The superprimary field $\Phi(z, \theta)$, is written as

$$
\Phi:=\Phi(z, \theta)=\varphi(z, \theta)+\theta \varphi_{1}(z)+\theta^{2} \varphi_{2}(z),
$$

where $\varphi_{1}(z)$ and $\varphi_{2}(z)$ are paragrassmann fields of grades 1 and 2, respectively. One can similarly define a complete superprimary field $\Phi(z, \bar{z}, \theta, \bar{\theta})$ with the weights $(\Delta, \bar{\Delta})$ and the expansion

$$
\Phi=\sum_{k, k^{\prime}=0}^{2} \theta^{k} \bar{\theta}^{k^{\prime}} \varphi_{k k^{\prime}},
$$

through (1) and (2), and obvious analogous relations with $\bar{L}_{n}$ 's and $\bar{G}_{r}$ 's. Now suppose that the first component field $\varphi(z)$ in chiral superprimary field $\Phi(z, \theta)$, has a logarithmic counterpart $\varphi^{\prime}(z)[20]$ :

$$
\left[L_{n}, \varphi^{\prime}(z)\right]=\left[z^{n+1} \partial_{z}+(n+1) z^{n} \Delta\right] \varphi^{\prime}(z)+(n+1) z^{n} \varphi(z) .
$$

We will show that $\varphi^{\prime}(z)$ is the first component field of a new superfield $\Phi^{\prime}(z, \theta)$, which is the formal derivative of the superfield $\Phi(z, \theta)$ with respect to its weight. Let us define the fields $f_{r}^{\prime}(z)$ by

$$
\left[G_{r}, \varphi^{\prime}(z)\right]=: z^{r+1 / 3} f_{r}^{\prime}(z),
$$

where $r+\frac{1}{3}$ is an integer. Following [22], acting on the both sides of the above equation with $L_{m}$ and using the Jacobi identity, and using (9), (1), and (5), we have

$$
\begin{aligned}
{\left[L_{m}, f_{r}^{\prime}(z)\right]=} & \left(\frac{m}{3}-r\right) z^{m}\left[f_{m+r}^{\prime}(z)-f_{r}^{\prime}(z)\right]+\left[z^{m+1} \partial_{z}\right. \\
& \left.+(m+1)\left(\Delta+\frac{1}{3}\right) z^{m}\right] f_{r}^{\prime}(z)+(m+1) z^{m} \varphi_{1}(z) .
\end{aligned}
$$

Demanding

$$
\left[L_{-1}, f_{r}^{\prime}(z)\right]=\partial_{z} f_{r}^{\prime}(z)
$$

it is easy to shown that

$$
f_{r}^{\prime}(z)= \begin{cases}\psi^{\prime}(z), & r \geq-1 / 3 \\ \psi^{\prime \prime}(z), & r \leq-4 / 3\end{cases}
$$

Then, equating $\left[L_{1}, f_{-4 / 3}^{\prime}(z)\right]$ and $\left[L_{1}, f_{-7 / 3}^{\prime}(z)\right]$, we obtain

$$
\psi^{\prime}(z)=\psi^{\prime \prime}(z)=: \psi_{1}^{\prime}
$$

So in this way we obtain a well-defined field $\psi_{1}^{\prime}$, satisfying

$$
\left[G_{r}, \varphi^{\prime}\right]=z^{r+1 / 3} \psi_{1}^{\prime}
$$




$$
\left[L_{n}, \psi_{1}^{\prime}\right]=\left[z^{n+1} \partial_{z}+(n+1) z^{n}\left(\Delta+\frac{1}{3}\right)\right] \psi_{1}^{\prime}+(n+1) z^{n} \psi_{1} .
$$

Again, let's define the fields $h_{r}^{\prime}(z)$ through

$$
\left[G_{r}, \psi_{1}^{\prime}\right]_{q^{-1}}:=-z^{r+1 / 3} h_{r}^{\prime}(z) .
$$

Acting both sides with $L_{m}$ and using the generalized Jacobi identity [18]:

$$
\left[\left[G_{r}, \psi_{1}^{\prime}\right]_{q^{-1}}, L_{m}\right]+\left[G_{r},\left[L_{m}, \psi_{1}^{\prime}\right]\right]_{q^{-1}}+\left[\left[L_{m}, G_{r}\right], \psi_{1}^{\prime}\right]_{q^{-1}}=0
$$

we obtain

$$
\begin{aligned}
{\left[L_{m}, h_{r}^{\prime}\right]=} & {\left[z^{m+1} \partial_{z}+(m+1)\left(\Delta+\frac{1}{3}\right) z^{m}\right] h_{r}^{\prime}+(m+1) z^{m} \psi_{2} } \\
& +\left(\frac{m}{3}-r\right) z^{m} h_{m+r}^{\prime}+\left(r+\frac{1}{3}\right) z^{m} h_{r}^{\prime} .
\end{aligned}
$$

Then, using the same method applied to determine the form of the functions $f_{r}^{\prime}(z)$, we find a well-defined field $\psi_{2}^{\prime}$ satisfying

$$
\begin{gathered}
{\left[G_{r}, \psi_{1}^{\prime}\right]_{q^{-1}}=-z^{r+1 / 3} \psi_{2}^{\prime}} \\
{\left[L_{n}, \psi_{2}^{\prime}\right]=\left[z^{n+1} \partial_{z}+(n+1)\left(\Delta+\frac{2}{3}\right) z^{n}\right] \psi_{2}^{\prime}+(n+1) z^{n} \psi_{2}}
\end{gathered}
$$

Finally, we must calculate $\left[G_{r}, \psi_{2}^{\prime}\right]_{q^{-2}}$. Substituting for $\psi_{2}^{\prime}(z)$ from (20) and (15), and using (6), we have

$$
\begin{aligned}
{\left[G_{r}, \psi_{2}^{\prime}\right]_{q}=} & -\left[z^{r+1 / 3} \partial_{z} \varphi^{\prime}(z)+(3 r+1) z^{r-2 / 3} \Delta \varphi^{\prime}(z)\right. \\
& \left.+(3 r+1) z^{r-2 / 3} \varphi(z)\right] .
\end{aligned}
$$

Now we define the quasi superprimary field $\Phi^{\prime}$ :

$$
\Phi^{\prime}:=\Phi^{\prime}(z, \theta)=\varphi^{\prime}(z)+\theta \psi_{1}^{\prime}(z)+\theta^{2} \psi_{2}^{\prime}(z) .
$$

It is easy to see that

$$
\begin{gathered}
{\left[L_{n}, \Phi^{\prime}\right]=\left[z^{n+1} \partial_{z}+(n+1) z^{n}\left(\Delta+\frac{\Lambda}{3}\right)\right] \Phi^{\prime}+(n+1) z^{n} \Phi} \\
{\left[G_{r}, \Phi^{\prime}\right]=\left[z^{r+1 / 3}\left(\delta_{\theta}-q^{2} \theta^{2} \partial_{z}\right)-(3 r+1) z^{r-2 / 3} q^{2} \Delta \theta^{2}\right] \Phi^{\prime}-q^{2}(3 r+1) z^{r-2 / 3} \theta^{2} \Phi}
\end{gathered}
$$

We see that (24) and (25) are formal derivatives of (1) and (2) with respect to $\Delta$, provided one defines the formal derivative [20-22]

$$
\Phi^{\prime}(z, \theta)=: \frac{d \Phi}{d \Delta} .
$$


The two superfields $\Phi$ and $\Phi^{\prime}$, are a two dimensional Jordanian block of quasiprimary fields. The generalization of the above results to an $m$ dimensional Jordanian block is obvious:

$$
\left[L_{n}, \Phi^{i}\right]=\left[z^{n+1} \partial_{z}+(n+1) z^{n}\left(\Delta+\frac{\Lambda}{3}\right)\right] \Phi^{i}+(n+1) z^{n} \Phi^{i-1},
$$

and

$$
\left[G_{r}, \Phi^{(i)}\right]=\left[z^{r+1 / 3}\left(\delta_{\theta}-q^{2} \theta^{2} \partial_{z}\right)-(3 r+1) q^{2} z^{r-2 / 3} \Delta \theta^{2}\right] \Phi^{i}-q^{2} \theta^{2}(3 r+1) z^{r-2 / 3} \Phi^{(i-1)} .
$$

Here $1 \leq i \leq m-1$, and the first member of the block, $\Phi^{(0)}$, is a superprimary field. It is easy to show that (27) and (28) are satisfied through the formal relation

$$
\Phi^{(i)}=\frac{1}{i !} \frac{d^{i} \Phi^{(0)}}{d \Delta^{i}}
$$

\section{Two point functions of Jordanian blocks}

Consider two Jordanian blocks of chiral quasi-primary fields $\Phi_{1}$ and $\Phi_{2}$, with the weights $\Delta_{1}$ and $\Delta_{2}$ and dimensions $p$ and $q$, respectively. As the only closed subalgebra of the super Virasoro algebra the central extension of which is trivial is formed by $\left[L_{-1}, L_{0}, G_{-1 / 3}\right]$, the correlator of fields with different weights may be nonzero. According to [18],

$$
\left.<\varphi_{k}^{(} 0\right) \varphi_{k^{\prime}}^{(0)}>=a_{K} \frac{A_{k k^{\prime}}\left(\Delta+\Delta^{\prime}+\left(k+k^{\prime}-3\right) / 3\right)^{B_{k k^{\prime}}}}{\left(z-z^{\prime}\right)^{\Delta+\Delta^{\prime}+\left(k+k^{\prime}\right) / 3}}=: a_{K} f_{k, k^{\prime}}\left(z-z^{\prime}\right),
$$

where $A_{k k^{\prime}}$ and $B_{k k^{\prime}}$ are the components of the following matrices:

$$
B=\left(\begin{array}{lll}
0 & 0 & 0 \\
0 & 0 & 1 \\
0 & 1 & 1
\end{array}\right), \quad A=\left(\begin{array}{ccc}
1 & 1 & 1 \\
-1 & 1 & 1 \\
q^{2} & -q^{2} & q^{2}
\end{array}\right),
$$

$a_{0}, a_{1}$, and $a_{2}$, are arbitrary constants, and

$$
K=k+k^{\prime} \bmod 3
$$

The general form of the two point functions of Jordanian blocks is then readily obtained, using (29):

$$
<\varphi_{k}^{(i)} \varphi_{k^{\prime}}^{(j)}>=\frac{1}{i !} \frac{1}{j !} \frac{d^{i}}{d \Delta^{i}} \frac{d^{j}}{d \Delta^{\prime j}} \frac{a_{K} A_{k k^{\prime}}\left(\Delta+\Delta^{\prime}+\left(k+k^{\prime}-3\right) / 3\right)^{B_{k k^{\prime}}}}{\left(z-z^{\prime}\right)^{\Delta+\Delta^{\prime}+\left(k+k^{\prime}\right) / 3}} .
$$


Here $0 \leq i \leq p-1$, and $0 \leq j \leq q-1$. In this formal differentiation, one should treat the constants $a_{i}$ as functions of $\Delta$ and $\Delta^{\prime}$. So, there will be other arbitrary constants

$$
a_{i}^{(j),(k)}:=\frac{d^{j}}{d \Delta^{j}} \frac{d^{k}}{d \Delta^{k}} a_{i}
$$

in these correlators.

To consider the correlators of the full field, one begins with

$$
<\varphi_{k \bar{k}}^{(00)}(z, \bar{z}) \varphi_{k^{\prime} \bar{k}^{\prime}}^{(00)}(z, \bar{z})>=a_{K \bar{K}} q^{-k \bar{k}} f_{k, k^{\prime}}\left(z-z^{\prime}\right) \bar{f}_{\bar{k}, \bar{k}^{\prime}}\left(\bar{z}-\bar{z}^{\prime}\right)
$$

obtained in [18]. Here $f_{k, k^{\prime}}\left(z-z^{\prime}\right)$ is defind in (30) and $\bar{f}_{\bar{k}, \bar{k}^{\prime}}\left(\bar{z}-\bar{z}^{\prime}\right)$ is the same as this with $\Delta \rightarrow \bar{\Delta}$ and $\Delta^{\prime} \rightarrow \bar{\Delta}^{\prime}$. Also,

$$
\begin{aligned}
& K=k+k^{\prime} \bmod 3 \\
& \bar{K}=\bar{k}+\bar{k}^{\prime} \bmod 3 .
\end{aligned}
$$

Using the obvious generalization of (29), it is easy to see that

$$
<\varphi_{k, \bar{k}}^{(i j)} \varphi_{k^{\prime}, \bar{k}^{\prime}}^{(l m)}>=\frac{1}{i ! j ! l ! m !} \frac{d^{i}}{d \Delta^{i}} \frac{d^{j}}{d \bar{\Delta}^{j}} \frac{d^{l}}{d \Delta^{\prime l}} \frac{d^{m}}{d \bar{\Delta}^{\prime m}}\left[a_{K \bar{K}} f_{k, k^{\prime}} \bar{f} \bar{k}, \bar{k}^{\prime}\right]
$$

Again, one should treat $a_{K \bar{K}}$ 's as formal functions of the weights, so that differentiating them with respect to the weights introduces new arbitrary parameters. 


\section{References}

[1] V. Gurarie; Nucl. Phys. B410 [FS](1993) 535

[2] H. Saleur; Yale preprint YCTP-P 38-91 (1991)

[3] L. Rozansky \& H. Saleur, Nucl. Phys. B376

[4] I.I. Kogan \& N.E. Mavromatos; Phys. Lett. B375 (1992) 461

[5] J. S. Caux, I.I. Kogan, A. Lewis, \& A. M. Tsvelik; Nucl. Phys. B489 (1997) 469

[6] I.I. Kogan \& A. Lewis; hep-th/9705240

[7] J. Cardy; J. Phys. A25 (1992) L201

[8] V. Gurarie, M. A. I. Flohr, \& C. Nayak; cond-math/9701212

[9] X. G. Wen, Y. S. Wu \& Y. Hatsugai; Nucl. Phys. B422 [FS] (1994) 476

[10] M. R. Rahimi Tabar \& S. Rouhani; Annals Phys. 246 (1996) 446

[11] M. R. Rahimi Tabar \& S. Rouhani; Nuovo Cimento B112 (1997) 1079

[12] M. R. Rahimi Tabar \& S. Rouhani; Europhysics. Lett. 37 (1997) 447

[13] M. A. I. Flohr; Nucl. Phys. B482 (1996) 567

[14] M. R. Rahimi Tabar \& S. Rouhani; Phys. Lett. A224 (1997) 331

[15] J. S. Caux, I. I. Kogan, \& A. M. Tsvelik; Nucl. Phys. B466 (1996) 444

[16] Z. Maassarani \& D. serban; Nucl. Phys. B489 (1997) 603

[17] A. M. Ghezelbash \& V. Karimipour; Phys. Lett. B402 (1997) 282

[18] F. Kheirandish \& M. Khorrami; hep-th/0004154

[19] A. T. Filippov, A. P. Isaev, \& A. B. Kurdikov; Int. J. Mod. Phys. A8 (1993) 4973

[20] M. R. Rahimi Tabar, A. Aghamohammadi, \& M. Khorrami; Nucl. Phys. B497 (1997) 555

[21] M. Khorrami, A. Aghamohammadi, \& M. R. Rahimi Tabar; Phys. Lett. B419 (1998) 179

[22] M. Khorrami, A. Aghamohammadi, \& A. M. Ghezelbash; Phys. Lett. B439 (1998) 283 